\title{
Practical Partial Decode and Forward ENCODING SCHEME FOR RELAY CHANNEL
}

\author{
Zouhair Al-qudah \\ Communication Engineering Department, Al-Hussein bin Talal University, \\ Ma'an, Jordan.
}

\begin{abstract}
In this paper, a source-destination pair, which is augmented by a half-duplex relay, is considered. Two practical partial decode and forward encoding schemes are proposed. In these transmission schemes, the relay may decode the source's signal either partially or completely. In each encoding technique, twophase transmission scheme is developed in which the relay can partially listen to the source in the first phase until it can generate the source's message and then, in the second phase, forward it to its destination. By employing these transmission phases, the achievable rates are obtained. Rigorous numerical examples are presented to $i)$ show the value of power allocation between the source and the relay, and ii) optimize the length of the listening phase.
\end{abstract}

\section{KEYWORDS}

Relay Channel, Power Allocation, Partial Decode and Forward.

\section{INTRODUCTION}

The three-node network, which is composed of a source-destination pair aided by a relay, is an effective solution in either extending or improving the signal reception. This basic network, which is commonly known as relay channel (RC), was extensively investigated in a wide range of different scenarios. For instance, the authors in [1]-[6] derived the achievable rates in the case that different encoding schemes are employed at the relay. These encoding techniques include decode and forward (DF) [1], [3], dynamic decode and forward [4], compute and forward [5], and partial decode and forward (PDF) [3], [6]. For example, in PDF, the relay is allowed to decode and then forward the public part of the source's message.

In practical communication scenarios, a given node cannot simultaneously receive and then transmit over the same frequency band. Thus, the RC with orthogonal channel components was studied in many different scenarios like [7], [8]. For instance, the authors in [7] derived the achievable rate in the case that the source transmits to both the relay and the destination over different frequency bands. Further, the transmission from the source and the relay to the destination is carried over the same frequency band. Another solution is to employ a half-duplex relay in which this intermediate node can listen to the source for a period of time, and then forward the signal into its destination in another time [9]-[11]. In this case, two main drawbacks can be identified. The first is that an inconvenience encoding scheme such as amplify-andforward is employed. The other drawback is that an inefficient spectral usage is obtained since full DF doubles the channel uses.

DOI: $10.5121 /$ ijwmn.2016.8405 
This paper proposes a new two-phase transmission encoding scheme in which the relay can partially decode the source's signal at the end of the first phase. Then, in the second phase, the relay can forward the source's signal into its destination. In particular, the achievable rate is derived in which a half-duplex relay is employed. This makes the source-relay channel and the relay-destination channel orthogonal in time. In addition, some numerical examples are shown to illustrate the importance of our theoretical work. For instance, the length of the first (listening) phase is optimized such that the achievable rate is maximized. Moreover, the optimum power allocation between the source and the relay is also numerically investigated.

The considered channel model is presented Section 2. Next, the achievable rate is derived in Section 3. Then, in Section 4, some numerical examples are studied to show the value of our theoretical results. Finally, the letter is concluded in Section 5.

\section{Physically DegRaded GaUSSiAn RELAY CHANNEL WITH HaLF-DUPLEX RELAY}

The RC is considered in which a source wants to communicate with its destination with an assistance of a relay. Specifically, the source wants to send a message, $W$, which is uniformly selected from $\mathcal{W} \in\left[1, e^{n R}\right]$, to its destination in $\mathrm{n}$ uses of the channel. This transmission can be performed with probability of error tends to 0 as the number of channel uses approaches infinity. Additionally, $\mathrm{R}$ is the achievable communication rate in nats. Moreover, the transmission is divided into two phases. In particular, in the first phase, which lasts for $m$ uses of the channel, the received signals, as shown in Fig. 1(a), by the relay, $Y_{R}$, and the destination, $Y_{D}$, are given as

$Y_{R}=h_{S R} X_{S}+Z_{R}$

$Y_{D}=h_{S D} X_{S}+Z_{D}$

where $X_{s}$ is the transmitted signal, constrained by an average power, $P_{s}$. In addition, $h_{: s K}$ and $h_{s D}$ are the source-relay and the source-destination channel gains, respectively. Throughout this letter, $Z_{R}$ and $Z_{D}$ are identically independent additive white Gaussian noise(AWGN) signals with zero mean and variances $N_{E}$ and $N_{D}$, respectively. In this encoding scheme, the relay is supposed to have knowledge regarding the code-book used by the source. At the end of this phase, the relay can decode the source's message and then generate the entire source's codeword. This process can enable this intermediate node to start forwarding to the destination in the second phase. Thus, the received signal by the destination, as depicted in Fig. 1(b), is expressed as

$Y_{D}=h_{S D} X_{S}\left\|h_{R D} X_{R}\right\| Z_{D}$

where $h_{n D}$ is the relay-destination channel gain. Indeed, since this phase can last for $(n-m)$ uses of the channel, thus, the average transmit power by the relay can be limited to $\frac{n}{n-m} P_{R}$.

\section{ACHIEVAble Rate OF RELAY ChanNel WITH PDF AT RELAY}

In this section, we derive the attainable rate of the three-node network in which the relay operates in half-duplex mode. In the first phase, the relay listens to the source until it can decode the source's signal and then generate the entire codeword. Then, the relay can forward the signal into 
its destination in the remaining available time period. We first introduce the signalling and the associated achievable rates.

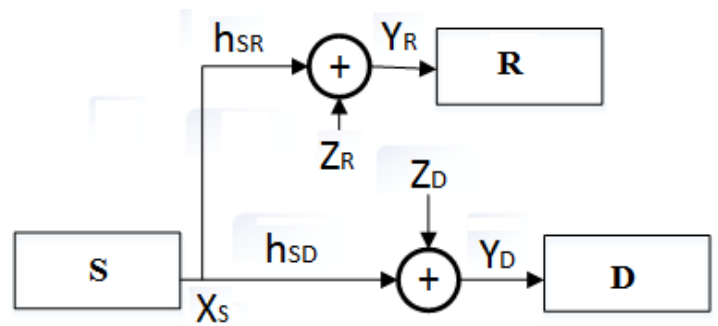

(a): Transmission in phase 1

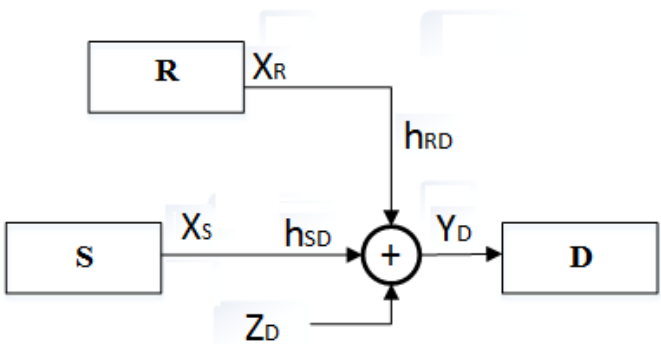

(b): Transmission in phase 2 .

Figure 1: Transmission Phases.

Phase-1: In this phase, the relay can hear the source signal, $X_{S}$ until it can generate the entire codeword. Therefore, the relay can observe the source's signal iff

$R_{S R} \leq \frac{m}{n} C\left(\frac{\left.h_{S R}\right|^{2} P_{S}}{N_{R}}\right)=I_{1}$

where $\quad C(x)=\frac{1}{2} \log _{\theta}(1+x)$.Moreover, this decoding process can be obtained with probability of error bounded as $P_{\theta} \leq e^{-m E(m G)}$ where $E($.$) is the Gallagher's random coding$ error exponent given as [12]

$E(m, G)=\max _{r \geq 1,0 \leq \sigma \leq 1} r G(1+\sigma)+\frac{1}{2} \log (1-2 r G)+\frac{\sigma}{2} \log \left(1-2 r G+\frac{G}{1+\sigma}\right)-\frac{n}{m} R_{S R}$

where $r$ and $\sigma$ are free parameters and needed to be optimized. $G=\frac{\left|h_{S R}\right|^{2} F_{S}}{N_{R}}$, which is the signal-to-noise ratio over the source-relay channel. Further, the relay receives a noisy version of $X_{S}$. Thus, the variance of this additional noise may be computed as

$\widehat{N}_{R}=P_{g} E\left[\left(X_{S}-Y_{R}\right)^{2}\right\rceil=2 P_{g} P_{S}$

In simple words, the relay uses the decoded symbols in the first phase to generate the remaining part of this signal. Then, in the second phase, the generated source's message is forwarded to its destination.

Phase-2: In this phase, both the source and the relay can transmit to their destination such that a multiple access channel is formed. Therefore, in this phase, the achievable rate can be expressed as 
International Journal of Wireless \& Mobile Networks (IJWMN) Vol. 8, No. 4, August 2016

$$
\begin{aligned}
R_{2} & =\frac{n-m}{n} I\left(X_{S}, X_{R}, Y_{D}\right) \\
& =\frac{n-m}{n}\left(h\left(Y_{D}\right)-h\left(Y_{D} \mid X_{S}, X_{R}\right)\right) \\
& =\frac{n-m}{n}\left(h\left(Y_{D}\right)-h\left(Z_{D}\right)\right) \\
& =\frac{n-m}{n} C\left(\frac{A_{n}}{V_{D}+\left|h_{R D}\right|^{n} \hat{N}_{R}}\right)
\end{aligned}
$$

where

$$
\begin{aligned}
& A_{2}=\left|h_{S D}\right|^{2} P_{S}+\frac{n}{n-m}\left|h_{R D}\right|^{2} P_{R}+2 \rho\left|h_{S D}\right|\left|h_{R D}\right| \sqrt{\frac{n}{n-m} P_{S}} P_{R} \\
& E\left[X_{S} X_{R}\right]=\rho\left|h_{S D}\right|\left|h_{K D}\right| \sqrt{\frac{n}{n-m} P_{S} P_{R}}
\end{aligned}
$$

At the end of this phase, the destination may start decoding the received signal. Now, we are ready to state the following Theorem.

Theorem: The Achievable rate, $\boldsymbol{R}$, of a source-destination pair aided by a half-duplex intermediate node (relay) can be expressed as

$$
R=\max _{m, P_{S}, P_{R}} \min \left(I_{2}+I_{3}, I_{4}\right)
$$

where

$$
\begin{gathered}
R_{1} \leq\left(\frac{m}{n}\right) C\left(\left|h_{s D}\right|^{2} P_{S}\right)=I_{2} \\
R_{2} \leq \frac{n-m}{n} C\left(\frac{A_{2}}{N_{D}+\left|h_{R D}\right|^{2} \tilde{N}_{R}}\right)=I_{3} \\
R_{1}+R_{2} \leq C\left(\frac{A}{N_{D}+\left|h_{R D}\right|^{2} N_{R}}\right)=I_{4}
\end{gathered}
$$

and $A=A_{2}+\left|h_{S R}\right|^{2} P_{S}$.

Proof: The proof can be easily obtained by following the established two-phase transmission scheme. In addition, the transmission in these two phases may form a multiple access channel [13] in which the sum rate is as given in $I_{4}$.

Remark-1: the following condition $I_{1}>I_{2}$ should be maintained for beneficial relaying. 
Remark-2: The achievable rate, $F$, gets its maximum in the case of $I_{2}+I_{3}=I_{4}$. In addition, in the case that the total available power is limited to $P_{T}$ such that $P_{T}=P_{S}+P_{R}$. Then, $P_{S}$ and $P_{R}$ cannot be analytical obtained in terms of $P_{T}$.

In the previous encoding scheme, the source is assumed to transmit over the whole $\mathrm{n}$ uses of the channel. However, in an another encoding technique, the source may be required to transmit only during the first phase. Consequently, the average power at the source is limited by $\frac{n}{m} P_{3}$. Moreover, the relay can transmit alone in the second phase. In this case, the achievable rate can be summarized by the following Corollary.

Corollary: The achievable rate of the RC with half-duplex relay, in which the source can completely transmit its signal in the first phase and the relay can transmit alone in the second phase, is given by

$$
R_{c}=\max _{m, P_{S}, P_{R}} \min \left(R_{3}, R_{4}\right)
$$

where

$$
\begin{aligned}
& R_{3}=\frac{m}{n} C\left(\frac{\frac{n}{m}\left|h_{s R}\right|^{2} P_{s}}{N_{R}}\right)+\frac{m}{n} c\left(\frac{\frac{n}{m}\left|h_{s D}\right|^{2} P_{s}}{N_{D}}\right) \\
& R_{4}={ }_{n}^{n-m} C\left(\begin{array}{c}
\frac{n}{n-m}\left|h_{R D}\right|^{2} P_{R} \\
N_{D}+\left|h_{R D}\right|^{2} \hat{N}_{R}
\end{array}\right)
\end{aligned}
$$

Since the source can restrict its transmission to only the first phase, thus, the relay may fully decode the source's message. In addition, this process can reduce the probability of error regarding of estimating the source's message at the relay.

Remark-3: We note that the probability of error decreases as the Gallager's exponent increases. Thus estimating the message signal at the relay in the case of full decoding, which is derived in the Corollary, is better than partial decoding scenario. Remember that, in full decoding, the relay may listen to the whole message, and the average power is increased to $\frac{n}{m} P_{s}$. Next, we numerically evaluate our theoretical analysis.

\section{NUMERICAL RESULTS}

In this section, some numerical examples are studied to show the optimal number of channel uses that the relay should listen to, and, the optimal allocated power to both the source and the relay. Unless otherwise specified, the channel gains $h_{S D}=1, h_{S R}=2$, and $h_{R D}=1.8$, are considered. Furthermore, the total available power $\Gamma_{S} \| \Gamma_{R}$ is set to 20. Hence, based on the channel gains $h_{S D}, h_{S R}$, and $h_{R D}$, the optimal numerical values of $P_{S}$ and $P_{R}$, are obtained. In addition, the variance of all noise signals is set to 1 . Finally, the total number of channel uses is set to 50 . 
Fig. 2 shows the achievable rate over the relay channel as a function of the first phase length, $m$, for different values of the channel gain $h_{E D}$. In particular, for a given value of $h_{R D}$, the achievable rate is a concave function of $m$. Noting that the maximum achievable rate over relay channel is obtained when both transmission phases have the same rate. Consequently, the value of $m$, at which the achievable rate reaches its maximum, determines the transition from the broadcast phase to multiple access phase. In addition, it is clear that the optimum value of $m$ varies with $h_{F D}$. For instance, for $h_{R D}=1.2$, the optimal value is $m=18$ whereas $m=23$ for $h_{R D}=2.4$. Furthermore, the associated allocated power to the relay is depicted in Fig. 3. In particular, this figure shows that more power is allocated to the relay as either $m$ increases or $h_{R D}$ decreases. For illustration, as $m$ increases, the relay has less number of channel uses to forward the source's signal. Therefore, more power is allocated such that the transmission rates during the two phases are equal.

Remark-4: Similar results to that shown in Fig. 2 can be easily obtained when the achievable rate, which is derived in the Corollary, is drawn versus $m$. In this case, the average power at the source is constrained $\frac{n}{m} P_{s}$. Accordingly, fewer number of channel uses, $m$, is required to estimate the source's signal.

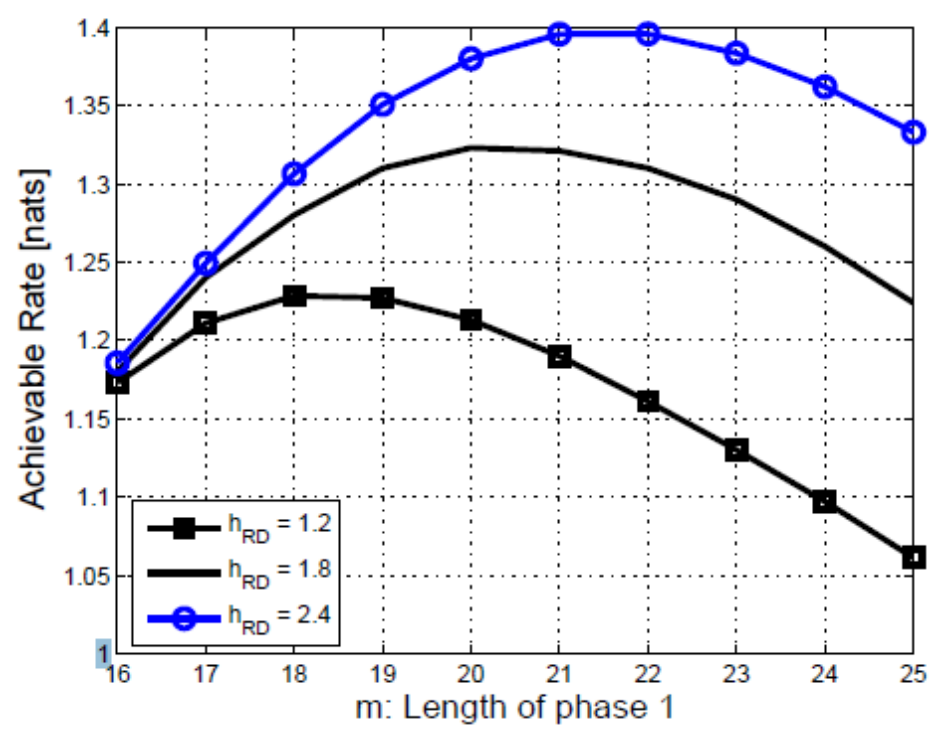

Figure 2. Achievable rate vs. $m$ for different values of the channel gain $h_{E D}$. 


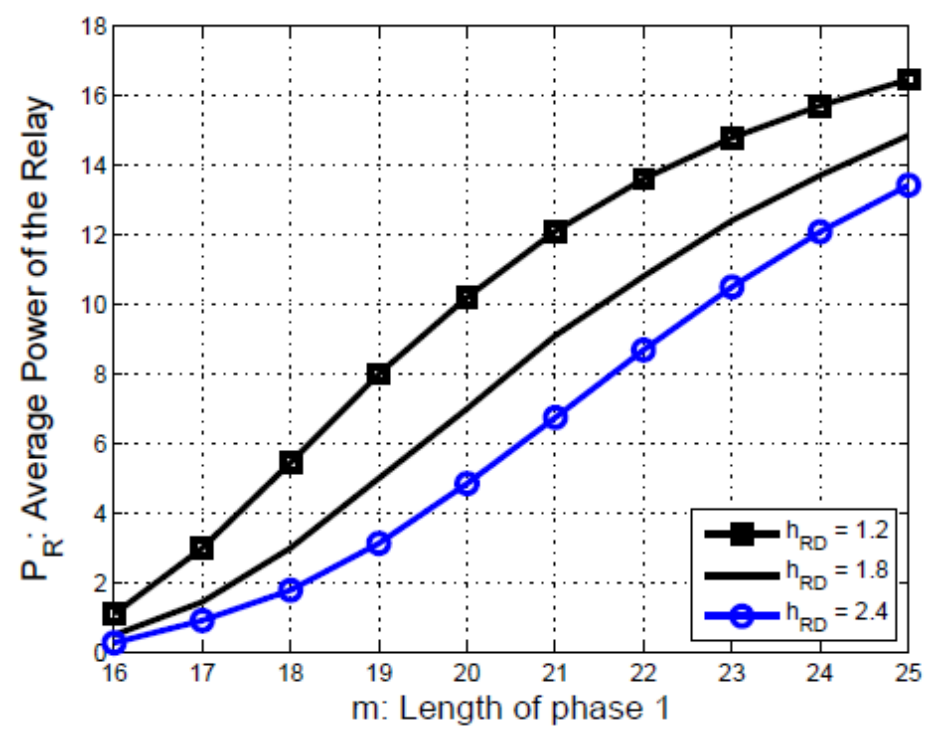

Figure 3. Allocated power to the relay vs. $m$ for different values of $h_{R D}$.

A comparison between the two derived achievable rates is carried out in Fig. 4. For instance, for value of $m=18$, the achievable rate, which is obtained in the Corollary, has higher rate than the other derived rate in the Theorem. The contrary is occurred for $m=23$. This is because the ultimate value of the achievable rate which is derived in the Corollary occurs at $m=\mathbf{1 8}$ whereas $m=23$ is the optimum value of the first phase length that maximize the rate derived in the Theorem.

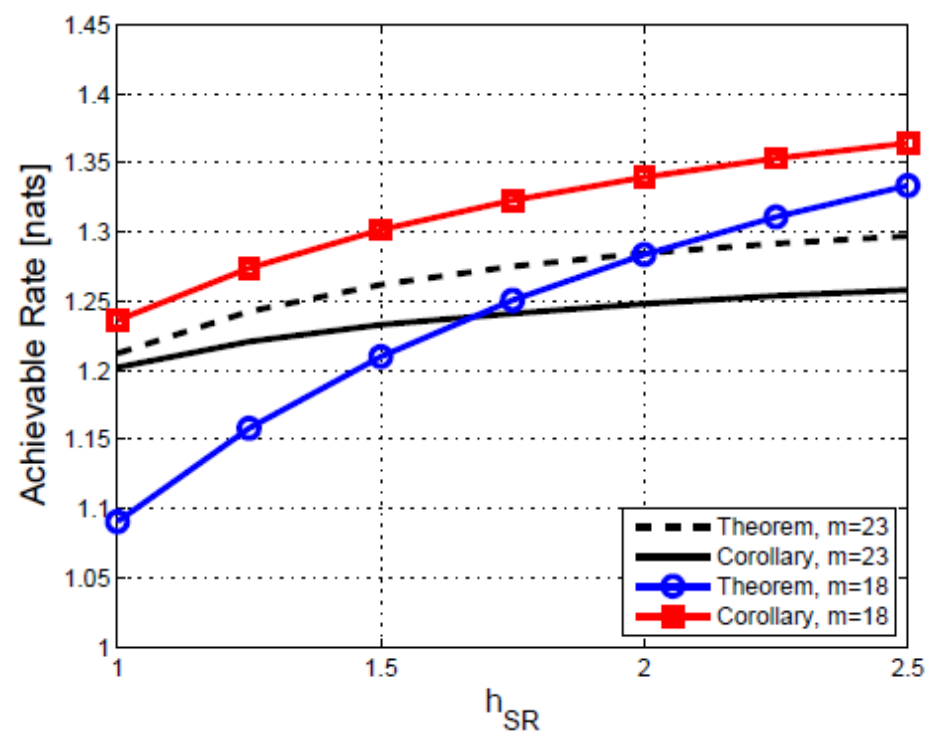

Figure 4. Comparison between the two derived achievable rates. 


\section{Conclusions}

The RC has been investigated in which the relay can operate in half-duplex mode. Two practical partial decode and forward encoding techniques have been developed. In each encoding scheme, the achievable rate has been derived when the relay can listen to the source until it can decode the source's signal. We have numerically studied the optimal power allocation that can maximize the achievable rates. In addition, the optimum duration of each transmission phase has been addressed.

\section{REFERENCES}

[1] T. Cover and A. Gamal, "Capacity theorems for the relay channel," IEEE Trans. Inf. Theory, vol. 25, pp. $572-584$, Sep. 1979.

[2] A. Gamal and M. Aref, "The capacity of the semi-deterministic relay channel (Corresp.)," IEEE Trans. Inf. Theory, vol. 28, pp. 536-536, May 1982.

[3] G. Kramer, M. Gastpar, and P. Gupta, "Cooperative Strategies and Capacity Theorems for Relay Networks," IEEE Trans. Inf. Theory, vol. 51, pp. 3037 - 3063, Sep. 2005.

[4] C.-P. Lee and H.-J. Su, "Dynamic Decode and Forward for the Multi-Access Relay Channel with Finite Block Length," in IEEE Int. Symp. Personal Indoor and Mobile Radio Communications (PIMRC), pp. 1825-1829, Sep. 2011.

[5] L. Wei and W. Chen, "Efficient Compute-and-Forward Network Codes Search for Two-Way Relay Channel,” IEEE Commun. Lett.,, vol. 16, pp. 1204-1207, Aug. 2012.

[6] P. Zhong and M. Vu, "Partial decode-forward coding schemes for the Gaussian two-way relay channel," in Int. Conference on Communications (ICC),, pp. 2451-2456, Jun. 2012.

[7] A. El Gamal and S. Zahedi, "Capacity of a class of relay channels with orthogonal components," IEEE Trans. Inf. Theory, vol. 51, pp. 1815-1817, May 2005.

[8] O. Sahin, O. Simeone, and E. Erkip, "Gaussian Interference Channel Aided by a Relay with Out-ofBand Reception and In-Band Transmission," IEEE Trans. Commun., vol. 59, pp. 2976-2981, Nov. 2011.

[9] T. Pham, H. Nguyen, and H. Tuan, "Power Allocation in Orthogonal Wireless Relay Networks With Partial Channel State Information,” IEEE Trans. Signal Process., vol. 58, pp. 869-878, Feb 2010.

[10] B. Rankov and A. Wittneben, "Spectral efficient protocols for half-duplex fading relay channels," IEEE J. Sel. Areas in Commun., vol. 25, pp. 379-389, Feb. 2007.

[11] F. Parzysz, M. Vu, and F. Gagnon, "A half-duplex relay coding scheme optimized for energy efficiency," in Information Theory Workshop (ITW), pp. 306-310, Oct 2011.

[12] R. Gallager, "A Simple Derivation of the Coding Theorem and Some Applications," IEEE Trans. Info. Theory, vol. 11, pp. 3 - 18, Jan. 1965.

[13] R. Nabar, H. Bolcskei, and F. Kneubuhler, "Fading relay channels: performance limits and space-time signal design,” IEEE J. Selec. Areas Commun., vol. 22, pp. 1099-1109, Aug. 2004. 